\title{
Trends in overall, cardiovascular and cancer-related mortality among individuals with diabetes reported on death certificates in the United States between 2007 and 2017
}

\author{
Donghee Kim ${ }^{1}$ • Andrew A. Li ${ }^{2}$. George Cholankeril ${ }^{1}$ - Sun H. Kim ${ }^{3}$ - Erik Ingelsson ${ }^{4,5}$ • Joshua W. Knowles ${ }^{4,5}$. \\ Robert A. Harrington ${ }^{2} \cdot$ Aijaz Ahmed ${ }^{1,5}$
}

Received: 21 February 2019 / Accepted: 14 March 2019 / Published online: 22 April 2019

(C) Springer-Verlag GmbH Germany, part of Springer Nature 2019

\begin{abstract}
Aims/hypothesis The determination of diabetes as underlying cause of death by using the death certificate may result in inaccurate estimation of national mortality attributed to diabetes, because individuals who die with diabetes generally have other conditions that may contribute to their death. We investigated the trends in age-standardised mortality due to diabetes as underlying or contributing cause of death and cause-specific mortality from cardiovascular disease (CVD), complications of diabetes and cancer among individuals with diabetes listed on death certificates in the USA from 2007 to 2017.

Methods Using the US Census and national mortality database, we calculated age-standardised mortality due to diabetes as underlying or contributing cause of death and cause-specific mortality rates among adults over 20 years with diabetes listed on death certificates. A total of 2,686,590 deaths where diabetes was underlying or contributing cause of death were analysed. We determined temporal mortality rate patterns by joinpoint regression analysis with estimates of annual percentage change (APC). Results Age-standardised diabetes mortality rates compared among underlying cause of death, contributing cause of death and all-cause mortality were 32.2 vs 75.7 vs 105.1 per 100,000 individuals during the study period. The age-standardised mortality rates due to diabetes as underlying or contributing cause of death declined from 112.2 per 100,000 individuals in 2007 to 104.3 per 100,000 individuals in 2017 with the most pronounced decline noted from 2007 to 2014 (APC $-1.4 \%$; 95\% CI $-1.9 \%$, $-1.0 \%$ ) and stabilisation in decline from 2014 to 2017 (APC 1.1\%; 95\% CI -0.6\%, 2.8\%). In terms of cause-specific mortality among individuals with diabetes listed on death certificates, the age-standardised mortality rates for CVD declined at an annual rate of $1.2 \%$ with a marked decline of $2.3 \%$ between 2007 and 2014. Age-standardised diabetes-specific mortality rates as underlying cause of death decreased from 2007 to 2009 (APC -4.5\%) and remained stable from 2009 to 2017. Agestandardised mortality rates for cancer steadily decreased with an average APC of $-1.4 \%$ (95\% CI $-1.8 \%,-1.0 \%)$ during the 11 -year period. Mortality in the subcategory of CVD demonstrated significant differences.

Conclusions/interpretation Current national estimates capture about $30 \%$ of all-cause mortality among individuals with diabetes listed as underlying or contributing cause of death on death certificates. The age-standardised mortality due to diabetes as underlying or contributing cause of death and cause-specific mortality from CVD in individuals with diabetes listed as underlying or contributing cause of death plateaued from 2014 onwards except for hypertensive heart disease and heart failure.
\end{abstract}

Keywords Cancer $\cdot$ Cardiovascular disease $\cdot$ Complication $\cdot$ Epidemiology $\cdot$ Mortality

Aijaz Ahmed

aijazahmed@stanford.edu

1 Division of Gastroenterology and Hepatology, Stanford University School of Medicine, 750 Welch Road no. 210, Palo Alto, CA 94304, USA

2 Department of Medicine, Stanford University School of Medicine, Stanford, CA, USA
3 Division of Endocrinology, Gerontology and Metabolism, Stanford University School of Medicine, Stanford, CA, USA

4 Cardiovascular Medicine and Cardiovascular Institute, Stanford University School of Medicine, Stanford, CA, USA

5 Stanford Diabetes Research Center, Stanford University School of Medicine, Stanford, CA, USA 


\section{Research in context}

\section{What is already known about this subject?}

- Diabetes is the tenth highest cause of death worldwide, and the number of deaths attributable to diabetes doubled from 1990 to 2010

- The current estimate of diabetes as the underlying cause of death may not be a reliable indicator of its actual contribution to national mortality

\section{What is the key question?}

- What are the recent trends in mortality due to diabetes as the underlying or contributing cause of death, and in causespecific mortality due to complications of diabetes, among individuals with diabetes listed on death certificates in the USA?

\section{What are the new findings?}

- Age-standardised all-cause mortality due to diabetes as the underlying or contributing cause of death and causespecific mortality from complications of diabetes among individuals with diabetes listed on death certificates declined $0.7-1.4 \%$ annually during the 11 year study period

- Cardiovascular disease- or diabetes-related mortality among individuals with diabetes listed on death certificates tended to decline earlier in the study period and then stabilise

How might this impact on clinical practice in the foreseeable future?

- These findings will encourage future studies with prospective longitudinal data to reconfirm our results and develop clinical algorithms to optimise the management of diabetes-related complications, in particular cardiovascular complications

\begin{abstract}
Abbreviations
APC Annual percentage change

CeVD Cerebrovascular disease

CVD Cardiovascular disease

HF Heart failure

HHD Hypertensive heart disease

IHD Ischaemic heart disease

NHIS National Health Interview Survey

NVSS National Vital Statistics System
\end{abstract}

\section{Introduction}

The prevalence of diabetes is rising rapidly across the world. Globally, the estimated age-standardised diabetes prevalence increased from $4.3 \%$ in 1980 to $9.0 \%$ in 2014 for men and from $5.0 \%$ to $7.9 \%$ in women [1]. In the USA, despite heightened awareness of diabetes-associated complications and concerted efforts to bring this national epidemic under control, the prevalence and incidence of diabetes have increased during recent decades [2]. Analyses using the National Health Interview Survey (NHIS) suggest a doubling of the incidence and prevalence of diabetes between 1990 and 2008, with a plateauing during 2008-2012 in the USA [3]. By 2015, an estimated 30.2 million adults aged $\geq 18$ years $(12.2 \%$ of all
US adults) had diabetes, of which 7.2 million (23.8\%) were not aware of or did not report having diabetes [4].

The Diabetes Atlas Group reported that 5.1 million deaths in 2013 were attributable to diabetes in the world [5]. The Global Burden of Disease Study reported that diabetes is ranked as the tenth highest cause of death worldwide, and the number of deaths attributable to diabetes doubled from 1990 to 2010 [6]. In the USA, diabetes has held steady as the seventh leading cause of death since 2006, according to the National Vital Statistics System (NVSS) [7, 8]. In 2015, based on the data from death certificates, the age-standardised diabetes-related mortality rate as the underlying cause of death was 21.3 per 100,000 individuals ( $2.9 \%$ of total deaths) in the USA [7]. However, the current estimate of diabetes as the underlying cause of death may not be a reliable indicator of its actual contribution to the national mortality [9]. It is well known that the most common cause of death among individuals with diabetes is cardiovascular disease (CVD) [10] Therefore, the information of death-certificate-assigned diabetes as an underlying cause of death is not sufficient for estimating national mortality attributed to diabetes [11-13], because individuals who die with diabetes generally have other conditions that may contribute to death (such as CVD, cancer, infectious disease, etc.).

There has been a marked decline in mortality from CVD due to improved treatment among the general population during recent decades $[14,15]$. A significant risk in mortality due 
to diabetes is attributed to CVD, but trends in mortality due to diabetes may be changing [16, 17]. Additionally, data outlining the trends in causes of death in individuals with diabetes in the USA are scarce. The Rochester Epidemiology Project showed no change in mortality from diabetes-related mortality and reduction in CVD-related mortality between 1970 and 1994 [18]. Recent advances in medical management of CVD such as effective therapy for dyslipidaemia, tighter control of BP and timely intervention with revascularisation may contribute to reduced CVD-related mortality among individuals with diabetes [19]. However, temporal trends in mortality for the subcategories of CVD including ischaemic heart disease, heart failure, hypertensive heart disease and cerebrovascular disease are lacking among individuals with diabetes in the USA. In this study, we used national mortality data from the NVSS to estimate recent trends in mortality rates due to diabetes as the underlying or contributing cause of death in the US general population from 2007 to 2017. Additionally, we investigated the mortality rates for CVD, cancer and diabetes among individuals with diabetes listed on death certificates in the USA between 2007 and 2017.

\section{Methods}

Study data To examine the trends in mortality rates due to diabetes as the underlying or contributing cause of death and cause-specific mortality rates among individuals with diabetes listed on death certificates in US adults aged $\geq 20$ years in the recent decade up to 2017, we analysed de-identified mortality records from the NVSS with death certificates as the main source of data. According to the instructions outlined by the NVSS, we downloaded the NVSS database for the death files and STATA input code files, which are available from the NVSS (https://www.cdc.gov/nchs/nvss/index.htm, accessed April 2018) and the National Bureau of Economic Research (http://www.nber.org/data/vital-statistics-mortality-datamultiple-cause-of-death.html, accessed April 2018). This mortality database captures over $99 \%$ of deaths in all states and the District of Columbia. Because the dataset for the population under 20 years was unreliable due to a small number of events, we excluded this population in our final analysis. In this dataset, cause of death was coded using the International Classification of Diseases, Tenth Revision (ICD10) (http://apps.who.int/classifications/icd10/browse/2016/ en). Each observation is one death with demographic characteristics [20]. The cause of death is incorporated into three fields: underlying cause of death, entity axis and record axis $[20,21]$. The underlying cause of death lists the one disease or condition that leads to death. The entity axis includes all causes of death listed on the death certificate. To promote specificity of the data or resolve contradictions, the record axis provides a refined version of the entity axis in which related conditions are combined for coding efficiency, overlapping conditions are eliminated and causes violating certain logical checks are deleted [21]. Because the record axis provides a dataset with higher specificity, we used the record axis for underlying or contributing causes of death listed on the death certificates to identify individuals with diabetes listed on death certificates [21]. Because NVSS datasets are de-identified and publicly available, our study did not require approval by the institutional review board.

Definitions of diabetes and cause-specific death Mortality due to diabetes (all-cause mortality) is the total death count of individuals who had diabetes listed as the underlying cause of death or contributing causes through the record axis. Causespecific mortality among individuals who had diabetes listed as an underlying or contributing cause of death was defined as the actual number of individuals who had CVD, cancer or accidents as an underlying cause of death. We defined the cause-specific mortality of diabetes as the actual number of individuals who had diabetes as an underlying cause of death irrespective of the status of contributing cause of death. Based on previous studies [10, 15], we used ICD-10 codes to identify all-cause mortality due to diabetes and cause-specific mortality among individuals with diabetes. All-cause mortality due to diabetes as underlying or contributing cause of death was defined as all deaths with underlying or contributing cause coded as E10-E14 using ICD-10 codes. Among individuals with diabetes listed on death certificates, CVD-related death was defined as ICD-10 I00-I99, cancer-related death as ICD-10 C00-C97 and diabetesrelated death as ICD-10 E10-E14 using the underlying cause of death. We categorised CVD-related death as ischaemic heart disease (ICD-10 I20-I25), hypertensive heart disease (ICD-10 I11 and I13), heart failure (ICD-10 I50) and cerebrovascular disease (ICD-10 I60-I69) using underlying cause of death [15]. Among individuals with diabetes listed on death certificates, accident-related death was defined as V01-X59 and Y85-Y86 using underlying cause of death. In addition, we analysed demographic information such as age, sex, race/ethnicity and education status based on the NVSS database. Age, sex, race/ethnicity and education status were ascertained using standard methods of the National Center for Health Statistics (https://www.cdc.gov/nchs/index.htm). Race/ethnicity was categorised into five mutually exclusive groups: non-Hispanic white, non-Hispanic black, nonHispanic Asian/Pacific Islander, non-Hispanic American Indian/Alaskan and Hispanic (who could be of any race).

Statistical analysis Age-specific mortality rates were calculated by dividing the number of deaths from diabetes as underlying or contributing cause of death or cause-specific deaths among individuals with diabetes listed on death certificates by 
the US Census population for each year from the US Census database. We calculated age-specific mortality rates per 100,000 individuals by age group $(20-29,30-39,40-49,50-59,60-69$, $70-79$ and $\geq 80$ years) based on the US Census database. Agespecific mortality rates were standardised to the age distribution of the 2010 US standard population using the direct method. We analysed our data using the 2000 and 2010 US standard populations for standardisation. There were no meaningful differences between the two except for higher age-standardised mortality rates for diabetes due to older age stratification in 2010 than in 2000. We described demographic characteristics of the study population as frequencies with percentages. To investigate temporal changes in trends over time, we used the National Cancer Institute's joinpoint regression (version 4.6.0.0, https://surveillance.cancer.gov/joinpoint/). This regression fits a series of combined straight lines to trend temporal data, which determines whether mortality rate is best explained by a single or several trend segments [22]. For each trend segment, the annual percentage change (APC) and the average APC, which is a summary measure of trend accounting for transitions within each trend segment, were calculated. Joinpoint regression calculates the year-to-year percentage change in annual age-standardised mortality rate and the 95\% CI over each trend segment [23].

\section{Results}

There was a total of 27,903,198 deaths among US adults aged $\geq 20$ years from 2007 to 2017 . The study population included 2,686,590 deaths (9.6\% of total) where diabetes was an underlying or contributing cause of death (all-cause mortality). CVD was the most common underlying cause of death and was associated with a third of the deaths, followed by diabetes and cancer (Table 1). As shown in Table 1, individuals who died from cancer as the underlying cause were more likely to be older than those who died from CVD or diabetes. Individuals who died from accident as the underlying cause were more likely to be younger than those who died from other causes. Among individuals with diabetes listed on death certificates, CVD was the leading cause of death in the non-Hispanic white and Asian groups, whereas in non-Hispanic black, non-Hispanic American Indian/Alaskan and Hispanic groups, diabetes ranked number one.

Trends in age-standardised mortality due to diabetes as the underlying or contributing cause of death and cause-specific mortality among individuals with diabetes reported on death certificates Figure 1 demonstrates age-standardised mortality due to diabetes as underlying or contributing cause of death and cause-specific mortality rates among individuals with diabetes reported on death certificates. The age-standardised mortality rates due to diabetes as underlying or contributing cause of death declined from 112.2 per 100,000 individuals in 2007 to 104.3 per 100,000 individuals in 2017 . Using joinpoint analyses (Table 2), the age-standardised mortality rates due to diabetes as underlying or contributing cause of death declined significantly from 2007 to 2014 (APC $-1.4 \%$; 95\% CI $-1.9 \%,-1.0 \%$ ) and then stabilised from 2014 to 2017 (APC $1.1 \%$; 95\% CI $-0.6 \%, 2.8 \%$ ). For cause-specific mortality among individuals with diabetes listed on death certificates, the age-standardised mortality rates for CVD declined annually by $1.2 \%$ with a marked decline at a rate of $-2.3 \%$ (95\% CI $-2.9 \%,-1.7 \%$ ) from 2007 to 2014 followed by stabilisation between 2014 and 2017 (Table 2). Similarly, the age-standardised diabetes-related mortality rates among individuals with diabetes reported on death certificates declined significantly during the earlier phase of the study period from 2007 to 2009 (APC $-4.5 \%$; 95\% CI $-7.9 \%,-0.9 \%$ ) and remained stable thereafter (APC $0.1 \%$; $95 \%$ CI $-0.3 \%$, $0.5 \%)$. Cancer-related mortality rates among individuals with diabetes listed on death certificates demonstrated a steady decline with an average APC of $-1.4 \%(95 \% \mathrm{CI}-1.8 \%,-1.0 \%)$. In contrast, accident-related mortality rates among individuals with diabetes reported on death certificates increased steadily with an average APC of 3.9\% (95\% CI 3.3\%, 4.5\%) during the 11-year study period. When we performed analyses to assess the mortality trends based on underlying cause of death and contributing cause of death, respectively (Fig. 2), we noted that the underlying cause of death captured about $30 \%$ of all-cause death among individuals who had diabetes listed as the underlying or contributing cause of death on their death certificates. Age-standardised diabetes-related mortality rates compared between underlying cause of death and mortality due to diabetes as underlying or contributing cause of death (all-cause mortality) were 32.2 vs 105.1 per 100,000 individuals ( 75.7 for contributing cause of death) during the study period. Comparable with all-cause mortality and diabetes-related death (as the underlying cause of death), age-standardised diabetes-related mortality rates based on contributing cause of death declined from 2007 to 2017 (APC $-0.6 \%$; 95\% CI $-1.4 \%, 0.1 \%$ ) with marginal significance (Table 2).

Trends in age-standardised aetiology-based CVD-related mortality among individuals with diabetes reported on death certificates Table 2 and Fig. 3 show the agestandardised mortality rates for subcategories of CVD among individuals with diabetes listed on death certificates. Agestandardised mortality rates for ischaemic heart disease declined significantly from 2007 to 2014 (APC $-2.8 \%$; $95 \%$ CI $-3.3 \%,-2.3 \%$ ) and then stabilised from 2014 to 2017 (APC $-0.6 \%$; 95\% CI $-2.5 \%, 1.3 \%$ ). Likewise, agestandardised mortality rates for cerebrovascular disease decreased from 2007 to 2014 (APC -3.1\%; 95\% CI $-4.1 \%$, 
Table 1 Characteristics of mortality due to diabetes as the underlying or contributing cause of death and cause-specific mortality among individuals with diabetes listed on death certificates in the USA, 2007-2017

\begin{tabular}{|c|c|c|c|c|c|}
\hline \multirow[t]{2}{*}{ Characteristic } & \multirow{2}{*}{$\begin{array}{l}\text { All-cause mortality } \\
\text { Mortality due to diabetes } \\
(n=2,686,590)\end{array}$} & \multicolumn{4}{|c|}{$\begin{array}{l}\text { Underlying cause of death among individuals with diabetes listed on death } \\
\text { certificates }\end{array}$} \\
\hline & & $\begin{array}{l}\text { CVD } \\
(n=895,530)\end{array}$ & $\begin{array}{l}\text { Cancer } \\
(n=296,230)\end{array}$ & $\begin{array}{l}\text { Diabetes } \\
(n=822,724)\end{array}$ & $\begin{array}{l}\text { Accident } \\
(n=46,846)\end{array}$ \\
\hline \multicolumn{6}{|l|}{ Age (years) } \\
\hline $20-39$ & $33,222(1.2)$ & $7065(0.8)$ & $761(0.3)$ & $16,869(2.1)$ & $1579(3.4)$ \\
\hline $40-59$ & $377,785(14.1)$ & $124,826(13.9)$ & $30,387(10.3)$ & $139,902(17.0)$ & 9067 (19.4) \\
\hline$\geq 60$ & $2,275,583(84.7)$ & $763,639(85.2)$ & $265,082(89.5)$ & $665,953(80.9)$ & $36,200(77.3)$ \\
\hline \multicolumn{6}{|l|}{ Ethnicity } \\
\hline White, non-Hispanic & $1,931,149(72.1)$ & $642,973(72.0)$ & $217,360(73.5)$ & $561,634(68.5)$ & $35,722(76.5)$ \\
\hline Black, non-Hispanic & $401,328(15.0)$ & $137,321(15.4)$ & $40,352(13.7)$ & $142,580(17.4)$ & $4796(10.3)$ \\
\hline $\begin{array}{l}\text { American Indian/Alaskan, } \\
\text { non-Hispanic }\end{array}$ & $26,160(1.0)$ & $7254(0.8)$ & $2333(0.8)$ & $9977(1.2)$ & $707(1.5)$ \\
\hline $\begin{array}{l}\text { Asian or Pacific Islander, } \\
\text { non-Hispanic }\end{array}$ & $78,073(2.9)$ & $27,684(3.1)$ & $9480(3.2)$ & $24,268(3.0)$ & $1438(3.1)$ \\
\hline Hispanic & $242,369(9.1)$ & $77,494(8.7)$ & $26,122(8.8)$ & $81,887(10.0)$ & $4010(8.6)$ \\
\hline \multicolumn{6}{|l|}{ Sex } \\
\hline Men & $1,415,393(52.7)$ & $490,499(54.8)$ & $167,701(56.6)$ & $432,495(52.6)$ & $25,564(54.6)$ \\
\hline Women & $1,271,197(47.3)$ & $405,031(45.2)$ & $128,529(43.4)$ & $390,229(47.4)$ & $21,282(45.4)$ \\
\hline \multicolumn{6}{|l|}{ Education } \\
\hline Less than high school & $555,843(26.1)$ & $181,950(25.6)$ & $57,100(24.2)$ & $172,499(26.3)$ & 8767 (23.4) \\
\hline Completed high school & $909,435(42.7)$ & $303,656(42.8)$ & $100,051(42.4)$ & $278,785(42.5)$ & $15,868(42.4)$ \\
\hline Some college & $263,741(12.4)$ & $88,566(12.5)$ & 30,385 (12.9) & $81,920(12.5)$ & $5008(13.4)$ \\
\hline Completed college or beyond & $402,033(18.9)$ & $135,871(19.1)$ & $48,284(20.5)$ & $122,514(18.7)$ & $7756(20.7)$ \\
\hline
\end{tabular}

We analysed mortality records from the National Vital Statistic System with death certificates as the main source of data between 2007 and 2017

Data are presented as the actual number of deaths from all-cause mortality (diabetes listed as the underlying or contributing cause of death) and causespecific death among individuals with diabetes listed on death certificates with stratification by age, ethnicity, sex and education groups in the columns. Percentage of total actual number (at the top of each column) is presented in parentheses (add up to 100 for each column) for each given group in each column. Ethnicity and education data were not available for some individuals because of limitations with death certificates

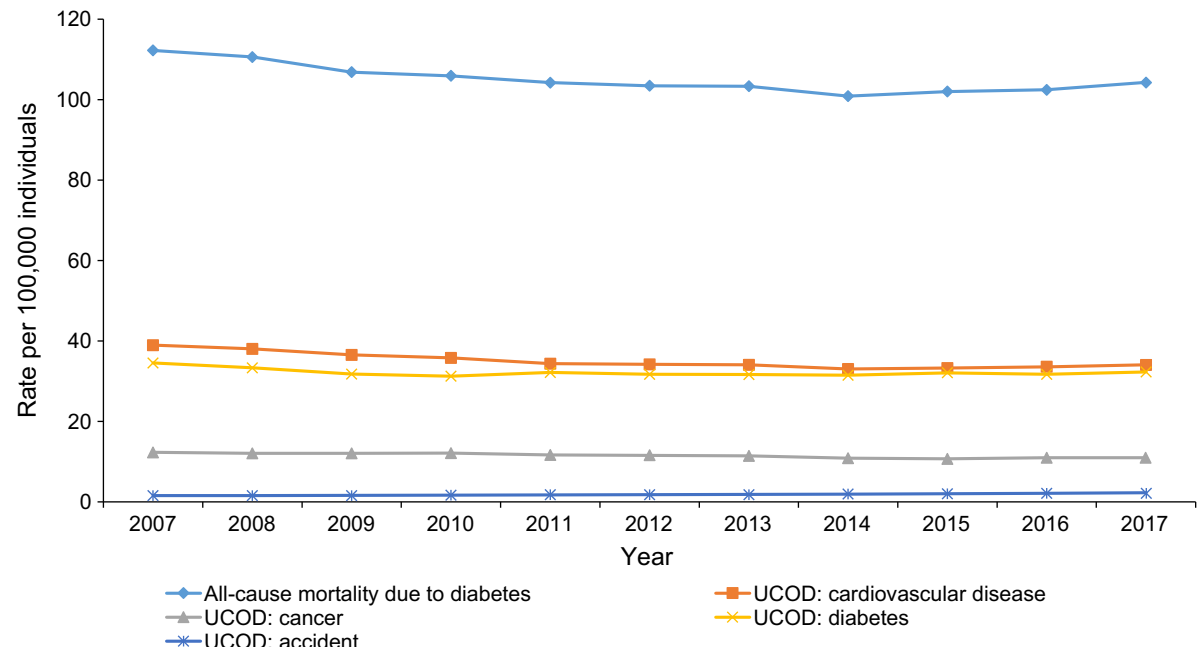

Fig. 1 Annual age-standardised all-cause mortality for diabetes as the underlying or contributing cause of death and cause-specific mortality rates among individuals with diabetes listed on death certificates in the USA, from 2007 to 2017 . We analysed mortality records from the NVSS with death certificates as the main source of data. We calculated age- specific mortality rates per 100,000 individuals by age group (20-29, $30-39,40-49,50-59,60-69,70-79$ and $\geq 80$ years) based on the US Census database and standardised to the age distribution of the 2010 US standard population using the direct method. UCOD, underlying cause of death 
Table 2 Age-standardised mortality rates due to diabetes as the underlying or contributing cause of death and cause-specific mortality rates among individuals with diabetes listed on death certificates and APC among US adults $\geq 20$ years, 2007-2017

\begin{tabular}{|c|c|c|c|c|c|c|c|}
\hline \multirow[t]{2}{*}{ Variable } & \multicolumn{2}{|c|}{ Age-standardised rate } & \multirow{2}{*}{$\begin{array}{l}\text { Average APC, \% (95\% CI) } \\
2007-2017\end{array}$} & \multicolumn{2}{|c|}{ Trend segment 1} & \multicolumn{2}{|c|}{ Trend segment 2} \\
\hline & 2007 & 2017 & & Year & $\mathrm{APC}, \%(95 \% \mathrm{CI})$ & Year & $\mathrm{APC}, \%(95 \% \mathrm{CI})$ \\
\hline \multicolumn{8}{|c|}{ All-cause mortality (diabetes as the underlying or contributing cause of death) } \\
\hline Mortality due to diabetes & 112.2 & 104.3 & $-0.7(-1.1,-0.2)^{*}$ & $2007-2014$ & $-1.4(-1.9,-1.0)^{*}$ & $2014-2017$ & $1.1(-0.6,2.8)$ \\
\hline \multicolumn{8}{|c|}{ Cause-specific death among individuals with diabetes listed on death certificates } \\
\hline CVD & 39.0 & 34.1 & $-1.2(-1.9,-0.6)^{*}$ & $2007-2014$ & $-2.3(-2.9,-1.7)^{*}$ & 2014-2017 & $1.3(-1.0,3.6)$ \\
\hline IHD & 23.4 & 18.7 & $-2.2(-2.7,-1.6)^{*}$ & 2007-2014 & $-2.8(-3.3,-2.3)^{*}$ & $2014-2017$ & $-0.6(-2.5,1.3)$ \\
\hline HHD & 2.3 & 3.4 & $3.8(2.7,4.8)^{*}$ & $2007-2014$ & $2.3(1.3,3.3)^{*}$ & $2014-2017$ & $7.3(3.4,11.3)^{*}$ \\
\hline $\mathrm{HF}$ & 1.7 & 1.7 & $-0.7(-1.8,0.4)$ & $2007-2011$ & $-4.4(-6.9,-1.8)^{*}$ & $2011-2017$ & $1.9(0.4,3.3)^{*}$ \\
\hline $\mathrm{CeVD}$ & 5.2 & 4.4 & $-1.6(-2.7,-0.6)^{*}$ & 2007-2014 & $-3.1(-4.1,-2.1)^{*}$ & 2014-2017 & $1.9(-1.9,5.8)$ \\
\hline Cancer & 12.3 & 11.0 & $-1.4(-1.8,-1.0)^{*}$ & 2007-2017 & $-1.4(-1.8,-1.0)^{*}$ & & \\
\hline Diabetes & 34.5 & 32.3 & $-0.8(-1.4,-0.2)^{*}$ & $2007-2009$ & $-4.5(-7.9,-0.9)^{*}$ & 2009-2017 & $0.1(-0.3,0.5)$ \\
\hline Accidents & 1.6 & 2.3 & $3.9(3.3,4.5)^{*}$ & 2007-2017 & $3.9(3.3,4.5)^{*}$ & & \\
\hline \multicolumn{8}{|l|}{ Contributing cause of death } \\
\hline Diabetes & 77.8 & 74.7 & $-0.6(-1.4,0.1)$ & $2007-2017$ & $-0.6(-1.4,0.1)$ & & \\
\hline
\end{tabular}

We calculated age-specific mortality rates per 100,000 individuals by age group $(20-29,30-39,40-49,50-59,60-69,70-79$ and $\geq 80$ years $)$ based on the US Census database and standardised to the age distribution of the 2010 US standard population using the direct method

We analysed mortality records from the NVSS with death certificates as the main source of data between 2007 and 2017

$p$ value for the APC and the average APC were calculated using joinpoint regression $\left({ }^{*} p<0.05\right)$

IHD, ischaemic heart disease; HHD, hypertensive heart disease; HF, heart failure; CeVD, cerebrovascular disease

$-2.1 \%)$ and then showed a relatively stable trend from 2014 to 2017 (APC 1.9\%; 95\% CI -1.9\%, 5.8\%). In contrast, agestandardised mortality rates for heart failure declined early in the study period from 2007 to 2011 (APC -4.4\%; 95\% CI $-6.9 \%,-1.8 \%$ ) but increased thereafter from 2011 to 2017
(APC $1.9 \%$; 95\% CI 0.4\%, 3.3\%). Mortality rates for hypertensive heart disease steadily increased, with a marked rise in APC from 2007 to 2014 (APC 2.3\%; 95\% CI 1.3\%, 3.3\%) and a steeper increase from 2014 to 2017 (APC 7.3\%; 95\% CI $3.4 \%, 11.3 \%)$.

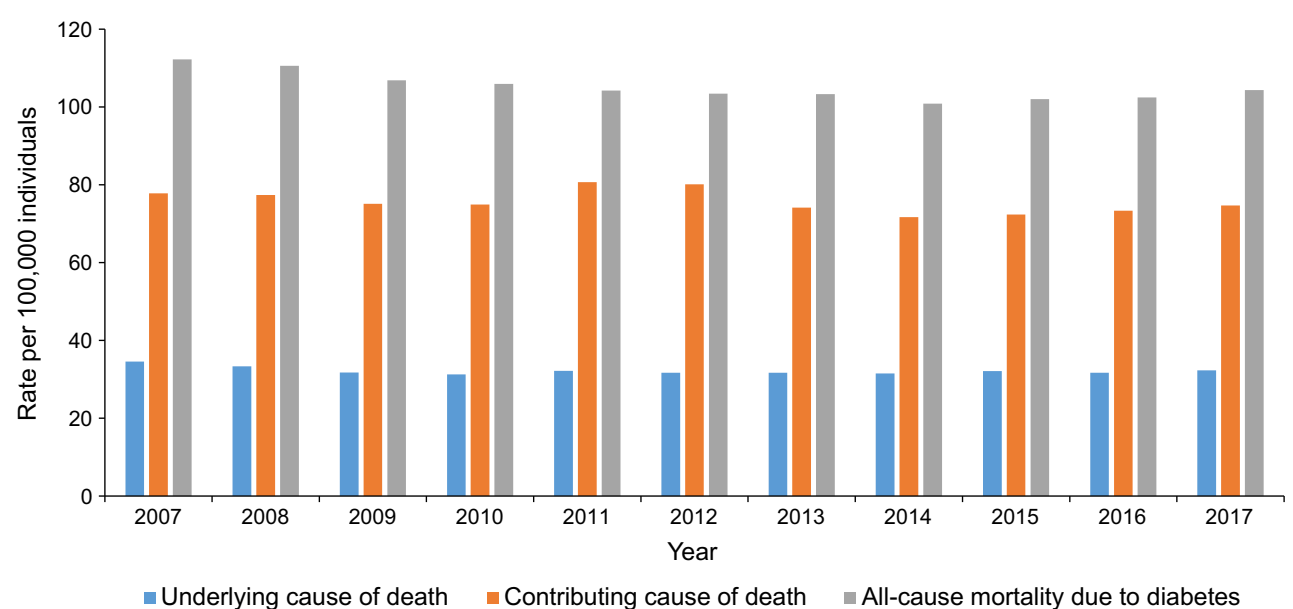

Fig. 2 Annual age-standardised all-cause mortality for diabetes as the underlying or contributing cause of death in the USA from 2007 to 2017. We analysed mortality records from the NVSS with death certificates as the main source of data. We calculated age-specific mortality

rates per 100,000 individuals by age group $(20-29,30-39,40-49,50$ $59,60-69,70-79$ and $\geq 80$ years) based on the US Census database and standardised to the age distribution of the 2010 US standard population using the direct method 


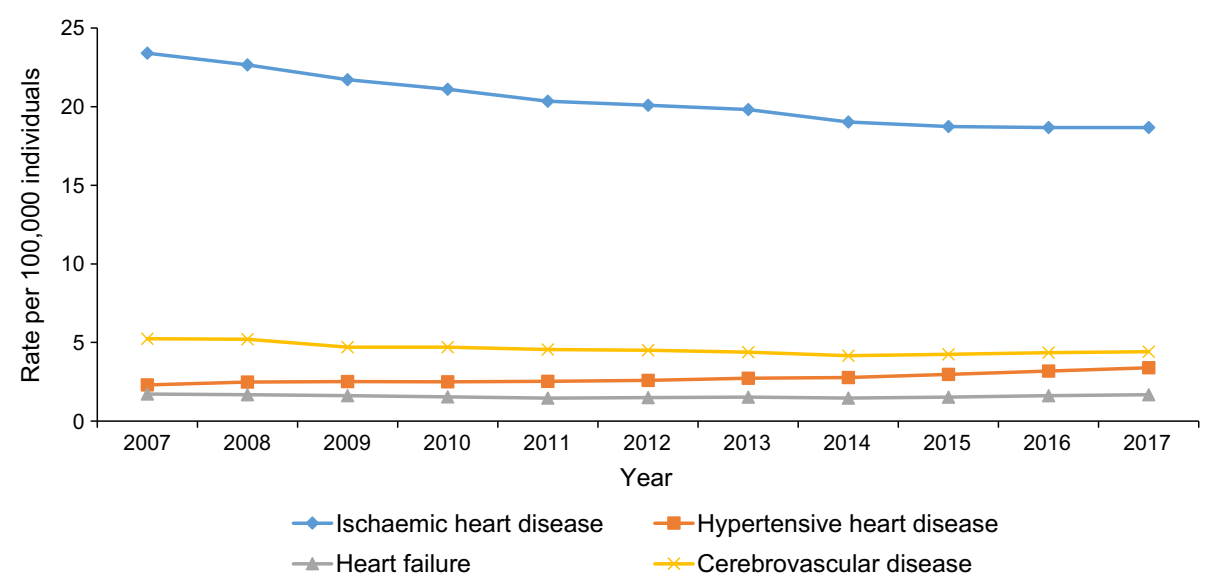

Fig. 3 Trends in aetiology-based annual age-standardised mortality rates for CVD among individuals with diabetes listed on death certificates in the USA, from 2007 to 2017 . We analysed mortality records from the NVSS with death certificates as the main source of data. We calculated

\section{Discussion}

In this population-based study using nationally representative mortality data from the USA, we demonstrate that the agestandardised all-cause mortality due to diabetes as the underlying or contributing cause of death and cause-specific mortality among individuals with diabetes listed on death certificates declined $0.7-1.4 \%$ annually during the 11 year study period. Rates in mortality due to diabetes as underlying or contributing cause of death and CVD or diabetes-related mortality among individuals with diabetes reported on death certificates tended to decline earlier in the study period and then stabilised. In terms of trends in mortality within the CVD subcategories, significant differences existed among individuals with diabetes listed on death certificates.

Our findings are consistent with previously reported trends in all-cause mortality due to diabetes and cause-specific mortality among individuals with diabetes [19, 24, 25]. Studies analysing diabetic cohorts from Framingham [26] and Minnesota [18] showed that diabetes-related mortality decreased during the 1990s. A study based on the US NHIS reported that death rates among subjects with diabetes declined between 1997 and 2006 [24]. A recently updated study using data from the NHIS reported age-specific mortality rates and proportional mortality from all causes, vascular causes, cancers, and non-vascular, non-cancer causes by diabetes status from 1985 to 2015 [27]. The mortality trends outlined in this study used cohorts with and without self-reported diabetes, which is the required study design for causes and trends in mortality regarding diabetes $[27,28]$. Consistent with our results, all-cause mortality rates declined $20 \%$ every 10 years among adults with diabetes, while death from vascular (32\%) and cancer-related $(16 \%)$ causes declined at a discordant rate. However, the decline in mortality rates was significantly greater among individuals with diabetes than those without age-specific mortality rates per 100,000 individuals by age group (20-29, $30-39,40-49,50-59,60-69,70-79$ and $\geq 80$ years) based on the US Census database and standardised to the age distribution of the 2010 US standard population using the direct method

diabetes [27]. In another study using the NHIS data, relative changes in 10-year mortality were significant for major CVD and ischaemic heart disease, but not in the heart failure subset, consistent with our analysis [29]. However, because these studies followed mortality until December of 2015, they were unable to exclude the possibility that the recent decline in diabetes-related death may be associated with the relatively short follow-up period in 2005-2009 (17.6 death rate per 1000 person-years) and 2010-2015 (15.2) compared with 1995-1999 (23.6) and 2000-2004 (21.4) subcohorts [27]. Our study demonstrates the declining trend in mortality due to diabetes as the underlying or contributing cause of death and CVD/cancer-related mortality among individuals with diabetes listed on death certificates regardless of a distinct gap in follow-up.

The improvement in outcomes observed in our study most likely reflects the impact of medical advances in the prevention, treatment and monitoring of this chronic disease. The decline in CVD-related mortality rates has been attributed to improvement in the management of risk factors related to CVD such as dyslipidaemia and hypertension, as well as advances in revascularisation techniques leading to better outcomes $[25,30]$. However, these findings have paradoxical implications for present and future burdens of diabetes in the USA. A declining mortality among individuals with diabetes in the past decade may have led to a substantial increase in the prevalence of diabetes and economic health burden related to diabetes [31, 32]. Interestingly, we found that all-cause mortality rates due to diabetes as the underlying or contributing cause of death and CVD-related mortality rates among individuals with diabetes listed on death certificates remained stable during the past 5 years. This may be partially explained by the excess mortality associated with diabetes being potentially spread among a considerably greater proportion of the population [24]. Additionally, reduction in mortality may lag 
behind improvement in the management and treatment of specific risk factors [24]. In real-life experience, such a lag time may result in the current plateau in mortality among individuals with diabetes [24]. One recent study suggests that individuals with diabetes in the USA had substantial improvements in control of risk factors between 1999 and 2010 [33]. However, $30-50 \%$ of individuals with diabetes still did not meet individual targets for glucose level, BP or lipid levels [33]. Another study demonstrated improvements in BP control and decline in the prevalence of smoking during the 2000s as compared with previous decades [34].

In our study, the mortality rate associated with hypertensive heart disease has increased among individuals with diabetes based on information listed on death certificates. Consistently, the mortality rate of heart failure has increased during recent years as well. This finding is somewhat unexpected because the rates of mortality for ischaemic heart disease have decreased during the study period. These observations suggest that other processes, perhaps less well-treated, which increase the risk of hypertensive heart disease and heart failure are not as affected by current clinical care best practices. Due to systemic inflammation, which anti-hypertensive medications do not alleviate, being normotensive on medication may not eliminate in its entirety the risks associated with hypertension among individuals with diabetes, especially insulin resistance or other comorbidities such as dyslipidaemia [35]. In our study, we noted that cancer-related mortality among individuals with diabetes listed on death certificates steadily declined between 2007 and 2017. A prospective cohort study, the Cancer Prevention Study-II, showed that diabetes was associated with a higher risk of certain malignancies including breast, liver and pancreas cancers [36]. In contrast to our results, a study from Australia reported that cancer-related mortality increased substantially between 1977 and 2010 [37]. In our study, the number of cancer-related deaths increased from 25,362 (age-standardised mortality rate: 12.3) in 2007 to 29,190 (age-standardised mortality: 11.0) in 2017; however, age-standardised mortality rates declined steadily during the study period. This discrepancy can be partly explained by the lack of age-standardised mortality rates in the Australian study. Consistent with our study, a recent study using the US NHIS data showed that deaths from cancers decreased $16 \%$ every 10 years from 1988-1994 to 2010-2015 [27].

Analyses of diabetes-related mortality, including current national reports from the NVSS, generally tend to include deaths for which diabetes was listed as the underlying cause of death. The availability of death-certificate-assigned diabetes as an underlying cause of death is not sufficient for estimating national mortality attributed to diabetes [11-13] because subjects with diabetes generally have other conditions that may directly contribute to death (such as CVD, cancer, renal disease, etc.). In our study, we analysed both agestandardised all-cause mortality due to diabetes as the underlying or contributing cause of death and cause-specific mortality rates among individuals with diabetes listed on death certificates. Mean age-standardised diabetes-related mortality rates compared between underlying cause of death and mortality due to diabetes as the underlying or contributing cause of death (all-cause mortality) were 32.2 vs 105.1 per 100,000 individuals ( 75.7 for contributing cause of death). In agreement with our study, a recent study using the NHIS dataset and the National Health and Nutrition Examination Survey demonstrated that the proportion of deaths attributable to diabetes (all-cause mortality) was estimated to be $11.5-11.8 \%$, while the proportion of deaths attributable to diabetes as underlying cause of death (3.3-3.7\%) underestimated the contribution of diabetes-related mortality in the USA [9]. Current annual estimates from the NVSS [38] are based on underlying cause of death, which may fail to capture diabetes-related mortality attributed to CVD, cancer and other causes of death.

The key strength of our nationally representative study is the reporting of longitudinal trends in aetiology-specific mortality rates for diabetes over an 11-year period. This allowed us to compare nationwide mortality data, thus gaining unique insight into mortality trends among individuals with diabetes listed on death certificates. We also used recently updated nationally representative mortality data, which enabled us to capture current trends for diabetes. Our main limitation is the definition of diabetes which is listed as the underlying or contributing cause of death on the death certificate. This study design and the dataset have the potential for underestimation and misclassification. Currently, robust data are lacking to validate the death certificate as a monitoring modality for death in diabetes. The Framingham Heart Study reported that death certificates were least accurate in individuals over 85 years and that there was no significant change in coding accuracy over time [39]. Coding method has been constant over time, so it is unlikely to account for the presented temporal trends in diabetes-related mortality rates. Furthermore, ICD-10 codes for diabetes and diabetes-related mortality grossly underestimate the true prevalence. A plausible explanation for the low rates of diabetes-related mortality is the significant underreporting on death certificates leading to underestimation of the true prevalence of diabetes by using ICD10 codes. This is an inherent limitation of the NVSS database. Such problems can be mitigated when analysing trends in which underestimation has been assumed to be relatively constant over the study period. Finally, age-standardised mortality rates may not represent actual mortality rates, but these rates were appropriate for comparisons during the study period as population distributions change over time.

In conclusion, age-standardised mortality due to diabetes as the underlying or contributing cause of death and causespecific mortality among individuals with diabetes listed on death certificates declined annually during recent decades. However, trends in all-cause mortality due to diabetes as the 
underlying or contributing cause of death and cause-specific CVD or diabetes-related mortality among individuals with diabetes listed on death certificates were noted to reach a plateau in 2010 and have remained stable thereafter. Our results suggest that greater efforts are needed to prevent mortality from CVD and complications associated with diabetes in the future.

Data availability National mortality datasets are publicly available at the National Vital Statistics System of the Center for Disease Control and Prevention (https://www.cdc.gov/nchs/nvss/index.htm).

Funding This research received no specific grant from any funding agency in the public, commercial or not-for-profit sectors. JWK was supported by the National Institutes of Health, USA (P30DK116074, U41HG009649, 1R01DK107437).

Duality of interest The authors declare that there is no duality of interest associated with this manuscript.

Contribution statement DK was responsible for study concept and design, acquisition of data, statistical analysis, interpretation of data, drafting of the manuscript, critical revision of the manuscript for important intellectual content and approval of the final draft manuscript. AAL, GC, SHK, EI, JWK and RAH were responsible for study concept and design, analysis and interpretation of data, critical revision of the manuscript for important intellectual content and statistical analysis, and approval of the final draft manuscript. AA was responsible for study concept and design, analysis and interpretation of data, critical revision of the manuscript for important intellectual content, approval of final draft manuscript and supervision of the research project. AA is responsible for the integrity of the work as a whole.

\section{References}

1. NCD Risk Factor Collaboration (2016) Worldwide trends in diabetes since 1980: a pooled analysis of 751 population-based studies with 4.4 million participants. Lancet 387:1513-1530

2. Cowie CC, Rust KF, Byrd-Holt DD et al (2006) Prevalence of diabetes and impaired fasting glucose in adults in the U.S. population: National Health And Nutrition Examination Survey 19992002. Diabetes Care 29(6):1263-1268. https://doi.org/10.2337/ dc06-0062

3. Geiss LS, Wang J, Cheng YJ et al (2014) Prevalence and incidence trends for diagnosed diabetes among adults aged 20 to 79 years, United States, 1980-2012. JAMA 312(12):1218-1226. https://doi. org/10.1001/jama.2014.11494

4. CDC NDSS (2015) National Diabetes Statistics Report. Available from https://www.cdc.gov/diabetes/pdfs/data/statistics/nationaldiabetes-statistics-report.pdf. Accessed 10 January 2018

5. IDF Diabetes Atlas Group (2015) Update of mortality attributable to diabetes for the IDF Diabetes Atlas: estimates for the year 2013. Diabetes Res Clin Pract 109(3):461-465. https://doi.org/10.1016/j. diabres.2015.05.037

6. Lozano R, Naghavi M, Foreman K et al (2012) Global and regional mortality from 235 causes of death for 20 age groups in 1990 and 2010: a systematic analysis for the Global Burden of Disease Study 2010. Lancet 380(9859):2095-2128. https://doi.org/10.1016/ S0140-6736(12)61728-0

7. Murphy SL, Xu J, Kochanek KD, Curtin SC, Arias E (2017) Deaths: final data for 2015. Natl Vital Stat Rep 66:1-75
8. Hunt BR, Whitman S, Henry CA (2014) Age-adjusted diabetes mortality rates vary in local communities in a metropolitan area: racial and spatial disparities and correlates. Diabetes Care 37(5): 1279-1286. https://doi.org/10.2337/dc13-0988

9. Stokes A, Preston SH (2017) Deaths attributable to diabetes in the United States: comparison of data sources and estimation approaches. PLoS One 12(1):e0170219. https://doi.org/10.1371/ journal.pone.0170219

10. Tancredi M, Rosengren A, Svensson AM et al (2015) Excess mortality among persons with type 2 diabetes. N Engl J Med 373(18): 1720-1732. https://doi.org/10.1056/NEJMoa1504347

11. Saydah SH, Geiss LS, Tierney E, Benjamin SM, Engelgau M, Brancati F (2004) Review of the performance of methods to identify diabetes cases among vital statistics, administrative, and survey data. Ann Epidemiol 14(7):507-516. https://doi.org/10.1016/j. annepidem.2003.09.016

12. Zhang X, Geiss LS, Cheng YJ, Beckles GL, Gregg EW, Kahn HS (2008) The missed patient with diabetes: how access to health care affects the detection of diabetes. Diabetes Care 31(9):1748-1753. https://doi.org/10.2337/dc08-0527

13. Fedeli U, Zoppini G, Goldoni CA, Avossa F, Mastrangelo G, Saugo M (2015) Multiple causes of death analysis of chronic diseases: the example of diabetes. Popul Health Metrics 13(1):21. https://doi.org/ 10.1186/s12963-015-0056-y

14. Global Burden of Cardiovascular Diseases Collaboration, Roth GA, Johnson CO et al (2018) The burden of cardiovascular diseases among US states, 1990-2016. JAMA Cardiol 3(5):375-389. https:// doi.org/10.1001/jamacardio.2018.0385

15. Rodriguez F, Hastings KG, Boothroyd DB et al (2017) Disaggregation of cause-specific cardiovascular disease mortality among hispanic subgroups. JAMA Cardiol 2(3):240-247. https:// doi.org/10.1001/jamacardio.2016.4653

16. Lind M, Garcia-Rodriguez LA, Booth GL et al (2013) Mortality trends in patients with and without diabetes in Ontario, Canada and the UK from 1996 to 2009: a population-based study. Diabetologia 56(12):2601-2608. https://doi.org/10.1007/s00125-013-3063-1

17. Ford ES (2011) Trends in the risk for coronary heart disease among adults with diagnosed diabetes in the U.S.: findings from the National Health and Nutrition Examination Survey, 1999-2008. Diabetes Care 34(6):1337-1343. https://doi.org/10.2337/dc102251

18. Thomas RJ, Palumbo PJ, Melton LJ 3rd et al (2003) Trends in the mortality burden associated with diabetes mellitus: a populationbased study in Rochester, Minn, 1970-1994. Arch Intern Med 163(4):445-451. https://doi.org/10.1001/archinte.163.4.445

19. Rawshani A, Rawshani A, Franzen S et al (2017) Mortality and cardiovascular disease in type 1 and type 2 diabetes. $\mathrm{N}$ Engl $\mathrm{J}$ Med 376(15):1407-1418. https://doi.org/10.1056/ NEJMoa1608664

20. Bureau USC (2017) The nation's older population is still growing, Census Bureau reports. Available at https://www.census.gov/ newsroom/press-releases/2017/cb17-100.html. Accessed 10 January 2018

21. Ly KN, Xing J, Klevens RM, Jiles RB, Ward JW, Holmberg SD (2012) The increasing burden of mortality from viral hepatitis in the United States between 1999 and 2007. Ann Intern Med 156(4): 271-278. https://doi.org/10.7326/0003-4819-156-4-20120221000004

22. Kim HJ, Fay MP, Feuer EJ, Midthune DN (2000) Permutation tests for joinpoint regression with applications to cancer rates. Stat Med 19(3):335-351. https://doi.org/10.1002/(SICI)10970258(20000215)19:3<335::AID-SIM336>3.0.CO;2-Z

23. Clegg LX, Hankey BF, Tiwari R, Feuer EJ, Edwards BK (2009) Estimating average annual per cent change in trend analysis. Stat Med 28(29):3670-3682. https://doi.org/10.1002/sim.3733 
24. Gregg EW, Cheng YJ, Saydah S et al (2012) Trends in death rates among U.S. adults with and without diabetes between 1997 and 2006: findings from the National Health Interview Survey. Diabetes Care 35(6):1252-1257. https://doi.org/10.2337/dc111162

25. Gregg EW, Gu Q, Cheng YJ, Narayan KM, Cowie CC (2007) Mortality trends in men and women with diabetes, 1971 to 2000. Ann Intern Med 147(3):149-155. https://doi.org/10.7326/00034819-147-3-200708070-00167

26. Preis SR, Hwang SJ, Coady S et al (2009) Trends in all-cause and cardiovascular disease mortality among women and men with and without diabetes mellitus in the Framingham Heart Study, 1950 to 2005. Circulation 119(13):1728-1735. https://doi.org/10.1161/ CIRCULATIONAHA.108.829176

27. Gregg EW, Cheng YJ, Srinivasan M et al (2018) Trends in causespecific mortality among adults with and without diagnosed diabetes in the USA: an epidemiological analysis of linked national survey and vital statistics data. Lancet 391(10138):2430-2440. https:// doi.org/10.1016/S0140-6736(18)30314-3

28. Bennett PH (2018) Diabetes mortality in the USA: winning the battle but not the war? Lancet 391(10138):2392-2393. https://doi. org/10.1016/S0140-6736(18)30843-2

29. Cheng YJ, Imperatore G, Geiss LS et al (2018) Trends and disparities in cardiovascular mortality among U.S. adults with and without self-reported diabetes, 1988-2015. Diabetes Care 41(11):23062315. https://doi.org/10.2337/dc18-0831

30. Ford ES, Ajani UA, Croft JB et al (2007) Explaining the decrease in U.S. deaths from coronary disease, 1980-2000. N Engl J Med 356(23):2388-2398. https://doi.org/10.1056/NEJMsa053935

31. Boyle JP, Thompson TJ, Gregg EW, Barker LE, Williamson DF (2010) Projection of the year 2050 burden of diabetes in the US adult population: dynamic modeling of incidence, mortality, and prediabetes prevalence. Popul Health Metrics 8(1):29. https://doi. org/10.1186/1478-7954-8-29

32. Carstensen B, Kristensen JK, Ottosen P, Borch-Johnsen K, Steering Group of the National Diabetes Register (2008) The Danish
National Diabetes Register: trends in incidence, prevalence and mortality. Diabetologia 51(12):2187-2196. https://doi.org/10. 1007/s00125-008-1156-z

33. Ali MK, Bullard KM, Saaddine JB, Cowie CC, Imperatore G, Gregg EW (2013) Achievement of goals in U.S. diabetes care, 1999-2010. N Engl J Med 368(17):1613-1624. https://doi.org/10. 1056/NEJMsa1213829

34. Wang J, Geiss LS, Cheng YJ et al (2011) Long-term and recent progress in blood pressure levels among U.S. adults with diagnosed diabetes, 1988-2008. Diabetes Care 34(7):1579-1581. https://doi. org/10.2337/dc11-0178

35. Rawshani A, Rawshani A, Franzen S et al (2017) Range of risk factor levels: control, mortality, and cardiovascular outcomes in type 1 diabetes mellitus. Circulation 135(16):1522-1531. https:// doi.org/10.1161/CIRCULATIONAHA.116.025961

36. Campbell PT, Newton CC, Patel AV, Jacobs EJ, Gapstur SM (2012) Diabetes and cause-specific mortality in a prospective cohort of one million U.S. adults. Diabetes Care 35(9):1835-1844. https://doi. org/10.2337/dc12-0002

37. Harding JL, Shaw JE, Peeters A, Guiver T, Davidson S, Magliano DJ (2014) Mortality trends among people with type 1 and type 2 diabetes in Australia: 1997-2010. Diabetes Care 37(9):2579-2586. https://doi.org/10.2337/dc14-0096

38. Heron M (2017) Deaths: leading causes for 2015. Natl Vital Stat Rep 66:1-76

39. Lloyd-Jones DM, Martin DO, Larson MG, Levy D (1998) Accuracy of death certificates for coding coronary heart disease as the cause of death. Ann Intern Med 129(12):1020-1026. https://doi.org/10.7326/0003-4819-129-12-199812150-00005

Publisher's note Springer Nature remains neutral with regard to jurisdictional claims in published maps and institutional affiliations. 